\title{
Fatores associados à morbimortalidade neonatal: um estudo de revisão
}

\author{
Graciela Gonsalves Borba1 Eliane Tatsch Neves² Andrea Moreira Arrué ${ }^{3}$ Andressa da Silveira ${ }^{4}$ Kellen Cervo Zamberlan ${ }^{5}$
}

\begin{abstract}
RESUMO
Objetivo - Identificar os fatores relacionados à morbimortalidade neonatal descritos na produção científica brasileira no período de 1989 a 2012. Método - Revisão integrativa realizada na Biblioteca Virtual de Saúde com descritores mortalidade neonatal and mortalidade infantil. Foram incluídos artigos de pesquisa com resumo em português disponível em suporte eletrônico. Compuseram o corpus da pesquisa 160 produções. Resultados - Os fatores para a morbimortalidade neonatal foram categorizados como relacionados ao neonato; gestante e gestação; acesso e qualidade do atendimento. Como principais fatores destacaram-se o baixo peso ao nascer, a prematuridade; as condições socioeconômicas, a idade materna; a qualidade da assistência ao pré-natal, ao parto e ao nascimento. Conclusão - Conclui-se que é necessário identificar esses fatores, oferecendo subsídios no intuito de minimizar os índices de morbimortalidade neonatal.
\end{abstract}

Descritores: Morbidade; Mortalidade Neonatal; Fatores de Risco; Enfermagem Pediátrica.

\section{Factors associated with neonatal morbidity and mortality: a review}

\begin{abstract}
Objective - To identify factors associated with neonatal mortality described in the Brazilian scientific production in the period 1989 to 2012. Method - Integrative review held at Virtual Health Library with descriptors neonatal mortality and infant mortality. We included research articles with abstracts in Portuguese available in electronic media. The organization of the data sample of 160 productions was described in a summary table. Results - The risk factors for neonatal morbidity and mortality were categorized as related to the neonate; pregnant and pregnancy, access and quality of care. Noteworthy is the low birth weight, prematurity, socioeconomic status, maternal age, quality of prenatal care, labor and birth. Conclusion - It is concluded that it is necessary to identify these factors, offering subsidies in order to minimize the rates of morbidity and mortality.
\end{abstract}

Descriptors: Morbility; Neonatal Mortality; Risk Factors; Pediatric Nursing.

\footnotetext{
${ }^{1}$ Graduada em enfermagem pela Universidade Federal de Santa Maria (UFSM), Santa Maria, RS, Brasil.

${ }^{2}$ Doutora em enfermagem pela Universidade Federal do Rio de Janeiro (UFRJ), Rio de Janeiro, RJ, Brasil.

${ }^{3}$ Mestre em enfermagem pela Universidade Federal de Santa Maria (UFSM), Santa Maria, RS, Brasil.

${ }^{4}$ Mestre em enfermagem pela Universidade Federal de Santa Maria (UFSM), Santa Maria, RS, Brasil.

${ }^{5}$ Mestranda em enfermagem pela Universidade Federal de Santa Maria (UFSM), Santa Maria, RS, Brasil.
} 


\section{Introdução}

A assistência neonatal progrediu no último decênio, principalmente pela evolução tecnológica vinculada à introdução de respiradores e a terapia intensiva neonatal bem como a terapia medicamentosa. Destaca-se a introdução de recursos terapêuticos mais eficazes e os recursos humanos especializados na área. ${ }^{1}$

A mortalidade neonatal está relacionada às condições de gestação, do parto e da própria integridade física da criança e a mortalidade pós-neonatal aparece vinculada às condições socioeconômicas e do meio ambiente, com predomínio das causas infecciosas. ${ }^{2}$ Os fatores de risco associados à morte neonatal precoce, tais como os nascimentos prematuros, baixo peso ao nascer e presença de intercorrências na gestação e no parto encontram-se descritos na literatura. ${ }^{3}$ No entanto, há uma rede complexa que envolve esses fatores, articulando características maternas e do Recém-Nascido (RN). Essas variáveis relacionadas desempenham um papel importante nas condições de nascimento e à saúde futura do RN.

Os índices de mortalidade perinatal e neonatal são indicadores sensíveis da adequação da assistência obstétrica e neonatal e do impacto dos programas de intervenção. Portanto, os níveis ainda elevados de mortalidade neonatal que ocorrem, no Brasil, apontam para a necessidade de melhor compreensão do papel da assistência no processo de determinação da saúde e da morbimortalidade neonatal. ${ }^{4}$

A iniciativa para redução da mortalidade infantil e fetal constitui-se uma estratégia importante para a compreensão das circunstâncias de ocorrência dos óbitos, identificação de fatores de risco e para definição das políticas de saúde dirigidas à sua redução. ${ }^{5}$ No Brasil, diversas iniciativas em nível estadual e municipal foram realizadas, cada qual de acordo com sua realidade, interesse e condição de operacionalização para redução desses índices.

Dessa forma, questionou-se: quais os principais fatores associados à morbimortalidade neonatal identificados na produção científica nacional nos últimos 22 anos?

Frente ao exposto, objetivou-se identificar os fatores relacionados à morbimortalidade neonatal descritos na produção científica brasileira no período de 1989 a 2012.

\section{Materiais e Métodos}

Revisão integrativa desenvolvida a partir da consulta no portal da Biblioteca Virtual em Saúde (BVS): na base de dados Literatura Latino-Americana e do Caribe em Ciências da Saúde (LILACS), Base de Dados de Enfermagem (BDENF) e portal Scientif Eletronic Library Online (SciELO). Utilizou-se a palavra mortalidade neonatal refinada com a palavra infantil and idioma português. Totalizou um universo de 3.336 artigos.

Foram incluídos artigos de pesquisa científica, pesquisa de campo ou bibliográfica publicada no período de 1989 a 2012. O recorte temporal teve como marco inicial a implantação do Sistema Único de Saúde (SUS) em 1988, considerandose, assim, os últimos 22 anos da produção científica. Sendo excluídos: teses, dissertações, manuais, notas prévias, reflexões teóricas, relatos de experiência, atualizações, publicações com resumos incompletos e/ou sem texto ou resumo indisponível online, e produções não convergentes com o objeto de estudo desta pesquisa. Foram selecionadas 253 publicações, destas 93 estavam repetidas em mais de uma base de dados. Portanto o corpus da pesquisa correspondeu a 160 produções.

A organização dos dados foi feita por meio de uma ficha documental com a seguinte informação: ano de publicação; subárea do conhecimento dos autores, região geográfica da produção; tipo de estudo; fatores associados à morbimortalidade neonatal. Para identificar os fatores de risco foi aplicada a análise de conteúdo temática. ${ }^{6} \mathrm{~A}$ análise dos estudos foi realizada de forma descritiva.

\section{Caracterização dos estudos:}

Em relação à região geográfica das produções encontradas destaca-se: $54,4 \%$ sudeste $(n=87), 20 \%$ sul ( $n=32), 18,1 \%$ nordeste $(n=29)$ e 4,4\% centro-oeste $(n=7), 1,2 \%$ norte $(n=2), 1,2 \%$ inter-regional $(n=2)$ e $0,6 \%$ internacional $(n=1)$.

Quanto às subáreas do conhecimento dos autores, identificou-se 60\% Medicina ( $n=96), 23,1 \%$ multiprofissionais $(n=37), 7,5 \%$ Enfermagem ( $n=12), 5,6 \%$ Epidemiologia ( $n=9)$. As subáreas, Estatística, Nutrição e Demografia com 1,2\% $(n=2)$ cada e 0,6\% Fisioterapia $(n=1)$. Importante salientar a restrita participação da Enfermagem nessas produções, embora participe de algumas publicações multiprofissionais. 
Quanto ao tipo de estudo, evidenciou-se $92 \%$ de pesquisas de campo $(n=147)$ e $8 \%$ de pesquisas bibliográficas $(n=13)$. Em relação ao ano de publicação dos artigos, tem-se que o primeiro artigo desta pesquisa foi publicado em 1989, evidenciando um aumento na frequência das produções nos períodos subsequentes, conforme o gráfico 1.

Verificou-se que o baixo peso ao nascer, a prematuridade e as malformações congênitas são os principais fatores de risco associados à morbimortalidade neonatal identificados no corpus desta pesquisa.

A literatura científica apresenta o baixo peso ao nascer (BPN) como principal fator de risco às condições de saúde e sobrevivência do neonatal. ${ }^{7-8} \mathrm{O}$ BPN se traduz em peso inferior à $2500 \mathrm{~g}$ ao nascimento, segundo ${ }^{9}$ que compreendem, 0 muito baixo peso e extremo baixo peso ao nascer como peso ao nascimento menor de $1500 \mathrm{~g}$ e $1000 \mathrm{~g}$, respectivamente.

Outras pesquisas evidenciaram as variáveis, BPN, prematuridade (PMT), malformações congênitas, baixo índice de Apgar e sexo masculino, como fatores prevalentes para o óbito neonatal..$^{8,10}$

Os problemas relacionados aos recém-nascidos de baixo peso e a mortalidade infantil (MI) quando comparados com crianças nascidas com peso adequado, evidenciam que o risco relativo de morte neonatal é quase 200 vezes maior nos com BPN. Prematuros de muito baixo peso estão mais vulneráveis à ocorrência de problemas de saúde ${ }^{11}$ incluindo ainda maior incidência para a mortalidade.

O fator de risco prematuridade (PMT) continua sendo um dos grandes dilemas da obstetrícia, respondendo por cerca de $70 \%$ das mortes neonatais. Esse fator está relacionado a elevados índices de morbidade, sendo responsável por metade das sequelas neurológicas, incluindo a paralisia cerebral. ${ }^{12}$

Estudo sobre corticoterapia na prevenção da síndrome de desconforto respiratório do recém-nascido (SDRRN) demonstrou a PMT como principal causa indireta de morte neonatal. ${ }^{13}$

O RN prematuro implica em cuidados especiais, devido a sua maior fragilidade orgânica e emocional.1 No que se refere ao tempo de vida do recém-nascido, pesquisa detectou que a maioria dos óbitos ocorreu na primeira semana de vida (92\%); em recém-nascidos com menos de 37 semanas de gestação (78,8\%); com BPN $(74,1 \%)$; do sexo masculino $(56,3 \%) .^{14}$

Já os neonatos com malformações congênitas, BPN, PMT e baixo índice de Apgar apresentaram maior incidência de óbito no período neonatal. ${ }^{15}$ As malformações congênitas foram mencionadas no geral, porém, alguns estudos citaram a onfalocele, hidrocefalia, macrossomia, gastrosquise e trissomias. O sexo masculino também foi mencionado como importante fator de risco para mortalidade neonatal. ${ }^{16}$ Conforme pesquisa desenvolvida em uma UTIN pública em Santa Maria/RS, no ano de 2006, 52\% dos óbitos foi do sexo masculino, ainda, neste mesmo estudo o índice de mortalidade estava diretamente associado a PMT. ${ }^{17}$

Pesquisa desenvolvida no município do Rio de Janeiro no período de 1979 a 2004 apontou uma TMI mais elevada entre os meninos, tanto no período neonatal quanto no pós neonatal. As afecções perinatais e as malformações congênitas também formam mencionadas como principais causas de óbito neonatais. ${ }^{18}$

Em relação às doenças infecciosas do neonato, os trabalhos pesquisados também salientaram a infecção neonatal (INN), sífilis congênita, candidíase sistêmica e o tétano neonatal. Este último apareceu em cinco estudos: um na região sul e quatro na região nordeste.

Em se tratando das afecções respiratórias, as mais constantes foram a SDRRN, em alguns estudos, ainda caracterizada, como doença da membrana hialina, bem como a insuficiência respiratória e infecção respiratória. No que se refere à asfixia perinatal, incluiu-se asfixia e anóxia como integrantes dessa variável. Sendo que todas essas afecções respiratórias decorrem de um outro fator pré-determinante que pode ser a PMT e/ou outras intercorrências relacionadas ao parto e nascimento.

\section{Fatores de risco para a morbimortalidade neonatal relacionados à gestante e a gestação}

O período gestacional é um fenômeno fisiológico, no entanto podem ocorrer agravos em sua evolução, colocando em risco a saúde da mãe e do bebê. ${ }^{19}$

Quanto aos fatores de risco encontrados neste estudo, destaca-se 19 relacionados à gestante e a gestação, sendo os expressos na Tabela 2 os que mais apareceram neste estudo. 
Tabela 2- Fatores de risco para a morbimortalidade neonatal relacionados à gestante e a gestação identificados na produção científica nacional entre 1989-2011. Brasil, RS 2013.

\begin{tabular}{c|c|c}
\hline Fator de risco & Frequencia absoluta (N) & Porcentagem (\%) \\
\hline Condições Socioeconomicas & 38 & 23,7 \\
\hline Idade Materna & 21 & 13,1 \\
\hline Idade Gestacional & 16 & 10 \\
\hline Doenças crônicas anteriores a gestação & 8 & 5 \\
\hline Menos de seis consultas de pré-natal & 5 & 3,1 \\
\hline Distúrbios específicos da gestação & 3 & 1,8 \\
\hline
\end{tabular}

As condições socioeconômicas bem como a idade materna e gestacional são apontadas na maioria dos estudos como principais fatores de risco para a morbimortalidade neonatal relacionados à gestante e a gestação.

No que se refere às condições socioeconômicas, foram englobados neste fator as variáveis relacionadas à renda familiar diminuída, ao grau de instrução materno, às mães sem companheiro, questão de extrema importância para a seguridade materna, bem como as condições de saneamento básico, características demográficas e distribuição espacial por região administrativa e bairro na cidade onde residem.

Estudo de caso-controle, realizado em Campinas/SP identificou como fatores associados ao óbito neonatal as variáveis nível socioeconômico e de condições da família, como renda, naturalidade e número de moradores no domicílio. ${ }^{20}$ Variáveis que refletem a exclusão social e fatores psicossociais, além dos fatores, BPN e PMT, também foram citados. ${ }^{3}$

A variável referente à idade materna foi abordada em 17 estudos, sendo que 10 produções referiram-se principalmente à gestação na adolescência, considerada pelos autores como a maternidade dos 13 aos 19 anos de idade. Outra variável abordada neste fator, porém com menor prevalência, diz respeito às mães com idade igual ou superior a 35 anos.

Estudo realizado no município do Rio de Janeiro/RJ, no período de 1996 e 1998, constatou o efeito expressivo da gestação na adolescência no baixo peso ao nascer. Foram utilizados dados disponíveis no Sistema de Informação sobre os Nascidos Vivos (SINASC), como o grau de instrução da parturiente e o tipo de maternidade procurada, se pública ou privada. ${ }^{21} \mathrm{~A}$ importância do controle da gestação na adolescência e também a priorização da atenção às mulheres sem companheiro ${ }^{22}$ corroboram resultados encontrados neste estudo.

Em São Luís/MA, as adolescentes apresentaram piores condições socioeconômicas e reprodutivas que as demais mulheres, as menores de 18 anos tiveram maiores proporções de filhos com BPN, PMT e com maior risco de mortalidade infantil. Já as adolescentes de 18 a 19 anos apresentaram resultados perinatais semelhantes às mulheres de 25 a 29 anos. ${ }^{23}$

Em contrapartida, em outra pesquisa o fator materno que se mostrou associado à mortalidade fetal e neonatal, na maioria dos estudos, foi a idade elevada. Determinantes como gestação na adolescência tiveram resultados conflitantes ou não significativos. ${ }^{24}$

Outro fator de risco relacionado à gestante e a gestação foram as doenças crônicas anteriores à gestação, que envolvem patologias como o diabetes melitus (DM), a cardiopatia, a obesidade e a hipertensão maternas. No que tange aos distúrbios específicos da gestação, destacam-se: o diabetes gestacional e a síndrome hipertensiva.

Estudo realizado com gestantes cardiopatas e diabéticas em São Paulo/SP trouxeram questões psicológicas da gestante em decorrência do fator de risco, onde a gestante experimenta o medo real em relação a si própria e ao seu filho, ao que está ocorrendo com seu corpo, ou ao temor de que seu filho nasça com anormalidades. ${ }^{25}$

Em estudo de caso controle realizado em Porto Alegre/RS, entre 2000-2003, que associou os óbitos evitáveis e nãoevitáveis e as variáveis sócio-demográficas, concluiu que a escolaridade materna menor ou igual a três anos; mãe sem companheiro ou com filhos nascidos mortos; malformação congênita; idade gestacional entre 22 e 36 semanas, peso inferior a $2.500 \mathrm{~g}$ e ter menos de seis dias de vida, apresentaram associação significativa com os óbitos evitáveis. ${ }^{26}$

Fatores de risco para a morbimortalidade neonatal relacionados ao acesso aos serviços de saúde e qualidade do atendimento

No que tange a saúde do neonato, observou-se ainda fatores de risco para a morbimortalidade associados ao acesso aos serviços de saúde e a qualidade do atendimento. Esta categoria detém um papel privilegiado na redução de 
complicações e óbitos neonatais. Sendo assim, para uma visão dimensionada dos fatores que se sobressaíram, eles serão apresentados na Tabela 3.

Nesta categoria agruparam-se cinco fatores de risco, dos quais três são apresentados na tabela acima por serem os mais frequentes nas produções científicas.

A qualidade do pré-natal prevaleceu, detendo um percentual de $16,2 \%$ das publicações. Foram incluídas neste fator, as variáveis referentes ao número de consultas, a realização ou não do pré-natal, além do conhecimento e habilidade do profissional que o realizou.

A qualidade da assistência ao parto e nascimento esteve presente em 13,7\% dos estudos e relaciona-se com as características do parto e nascimento como o local onde estes ocorreram e a própria qualidade da assistência prestada, capacitação dos profissionais, estrutura do local, e atendimento em tempo adequado.

Pesquisa que comparou a distribuição e a frequência de mortes reduzíveis em três municípios brasileiros, do Paraná, constatou a influência das condições sociais na determinação do acesso aos serviços e à sua utilização. Em uma das três cidades, $59,2 \%$ dos óbitos perinatais foram classificados como reduzíveis, principalmente por falhas na assistência ao parto e ao RN. ${ }^{27}$

Ao analisarem-se os óbitos infantis, segundo variáveis relacionadas à criança, à mãe e assistência em Cianorte/PR, no período de 1999 a 2006, detectou-se que 6,8\% das mães não fizeram nenhuma consulta de pré-natal, 95,5\% inseriramse em ocupações de baixa qualificação, $80,7 \%$ dos óbitos ocorreram no período neonatal, $68,3 \%$ por causas perinatais, $61,4 \%$ das crianças eram prematuras e $70,5 \%$ dos óbitos formam considerados evitáveis. ${ }^{28}$

A qualidade do pré-natal é um importante fator de consideração, um estudo transversal descritivo de avaliação dos serviços de saúde em Pelotas/RS, encontrou baixa proporção de adequação de pré-natal (5\%), resultado que, segundo os autores, sofreu influência do perfil das gestantes estudadas, em sua maioria de baixa renda. ${ }^{29}$

A dificuldade no acesso ao pré-natal ainda é um problema, como indicou estudo realizado em maternidades do Recife/ $P E$ no qual essa foi uma das principais justificativas apontadas pelas mães para a não-realização dessas consultas. ${ }^{30}$

Os óbitos relacionados a problemas maternos e que dependem de uma boa qualidade da atenção ao pré-natal e ao parto foram os de maior importância na mortalidade neonatal apontados em instituição terciária do estado de Pernambuco, entre 2001 e 2003, a partir dos dados das declarações de nascimento e óbito. ${ }^{31}$

Assim, considera-se relevante um olhar atentivo para os fatores de risco relacionados ao acesso e a qualidade da assistência à mulher, desde o planejamento familiar até a gestação, visto que esse é um fator que depende, principalmente, do Estado e dos profissionais de saúde envolvidos no processo.

\section{Conclusões}

O estudo constatou que o maior número de produções relaciona-se a pesquisas de campo de natureza clínicoepidemiológica, com predominância da região sudeste, e maior prevalência no ano de 2005. As produções analisadas foram categorizadas quanto aos fatores para a morbimortalidade neonatal destacando-se os relacionados ao neonato, a gestante e a gestação e, ainda, ao acesso e qualidade do atendimento. Dentre a primeira, destaca-se o baixo peso ao nascer e a prematuridade; na segunda as condições socioeconômicas e a idade materna; e na terceira a qualidade do pré-natal e a qualidade da assistência ao parto e ao nascimento.

A assistência ao pré-natal e ao parto tem um papel privilegiado na redução de complicações e óbitos neonatais e pode ser vista como uma política compensatória, cabendo-lhe o papel de minimizar o efeito das desigualdades socioeconômicas. Nesse sentido, observa-se que as taxas de mortalidade perinatal no Brasil são ainda elevadas, a maioria dos óbitos são considerados evitáveis e que poderiam ser prevenidos por meio de políticas que visem à promoção e a prevenção dos agravos à saúde.

Conclui-se que a atuação dos enfermeiros pode contribuir significativamente na identificação desses fatores, possibilitando um acompanhamento durante o pré-natal, parto e nascimento de qualidade, para a melhoria da assistência e a garantia de acesso mediante políticas públicas na área da saúde materno-infantil. 


\section{Referências bibliográficas}

1. Morais AC, Quirino MD, Almeida MS. O cuidado da criança prematura no domicílio. Acta paul enferm. 2009; 22(1):24-30. 2. Caldeira AP, Françab E, Perpétuo IHO, Goulart EMA. Evolução da mortalidade infantil por causas evitáveis, Belo Horizonte, 1984-1998. Rev saúde pública. [Periódico da internet]. 2005 [citado em 2012 Dez 12];39(1):67-74.

3. Schoeps D, Almeida MF, Alencar GP, França Júnior I, Novaes HMD, Arnaldo Siqueira AAF et al. Fatores de risco para mortalidade neonatal precoce. Rev. saúde pública. [Periódico na Internet]. 2007 [citado 2012 Dez 10]; 41(6): 1013-22. Disponível em: http://www.scielo.br/pdf/rsp/v41n6/6007.pdf.

4.. Maran E, Uchimura TT. Mortalidade Neonatal: fatores de risco em um município no sul do Brasil. Rev. Eletr. Enf. [Internet]. 2008;10(1):29-38. Available from: http://www.fen.ufg.br/revista/v10/n1/v10n1a03.htm

5. Ministério da Saúde (BR). Secretaria de Atenção à Saúde. Departamento de Ações Programáticas Estratégicas. Manual dos comitês de prevenção do óbito infantil e fetal. [Online]. Brasília: Ministério da Saúde, 2004. 60p. [citado 2012 Jan 12]. Disponível em: http://bvsms.saude.gov.br/bvs/publicacoes/MS_manual_finalizadoOBITOS.pdf.

6. Minayo MCS. O desafio do conhecimento: pesquisa qualitativa em saúde. 11a. ed. São Paulo: Hucitec; 2008.

7. Brasil. Ministério da Saúde. Secretaria de Vigilância em Saúde. Departamento de Análise de Situação em Saúde. Saúde Brasil 2006: uma análise da situação de saúde no Brasil. Brasília: Ministério da Saúde; 2006. 620p.

8. Ribeiro AM, Guimarães MJ, Lima MC, Sarinho SW, Coutinho SB. Fatores de risco para mortalidade neonatal em crianças com baixo peso ao nascer. Rev saúde pública. [Periódico na internet]. 2009 [Citado 2011 Nov 22]; 43(2):246-55. Disponível em: http://www.scielo.br/scielo.php?pid=S0034-89102009005000004\&script=sci_arttext.

9. Leone CR, Costa MTZ, Kahhale S. Mortalidade Perinatal e Neonatal. In: Marcondes E. Pediatria Básica Tomo I Pediatria geral e Neonatal. $9^{a}$ ed. São Paulo: Sarvier; 2003. 843p.

10. Tiago LF, Caldeira AP, Vieira MA. Fatores de risco de baixo peso ao nascimento em maternidade pública do interior de Minas Gerais. Pediatria. 2008;30(1):8-14.

11. Tronchin DMR, Tsunechiro MA. Prematuros de muito baixo peso: do nascimento ao primeiro ano de vida. Rev gaúch enferm. [Periódico na internet]. 2007 [Citado 2011 Nov 22];28(1):79-88. Disponível em: http://seer.ufrgs.br/ RevistaGauchadeEnfermagem/article/view/4703/2621.

12. Alencar Júnior CA, Feitosa FEL, Carvalho FHC, Ribeiro GG, Barreto JA, Alencar MFMF et al. Intervenções antenatais para redução da morbimortalidade neonatal devido a prematuridade. Femina. 2005;33(2):127-34 .

13. Andrade CRA, Guimaraes LM, Bracco MDA, Galletta MAK. Corticoterapia na prevenção da síndrome de desconforto respiratório do recém-nascido. Rev med. 1997; 76(4): 208-20.

14. Bercini L, Vianna LAC. A mortalidade neonatal de residentes em Maringá- Paraná. Rev ciênc cuid saúde. 2002;1(1):2934.

15. Helena ETS, Sousa CA, Silva CA. Fatores de risco para mortalidade neonatal em Blumenau, Santa Catarina: linkage entre bancos de dados. Rev bras saúde matern infant. [Periódico na internet]. 2005 [Citado 2011 Nov 22]; 5(2):09-17. Disponível em: http://www.scielo.br/pdf/rbsmi/v5n2/a10v05n2.pdf.

\section{Andréia Moreira Arrué}

Endereço correspondência - Toledo Piza, n³7. Bairro São João, CEP 27253-250, Volta Redonda, RJ, Brasil.

E-mail: andrea.mor@hotmail.com

Currículo Lattes: http://lattes.cnpq.br/0286094906805706

Graciela Borba - gracielaborba@yahoo.com.br

Eliane Tatsch Neves - elianeves03@gmail.com

Andressa da Silveira - andressadasilveira@gmail.com

Kellen Cervo Zamberlan - kellencz@hotmail.com

Recebido em 31 de janeiro de 2013.

Publicado em 07 de novembro de 2013. 